G. Plewig

T. Jansen

Department of Dermatology,

Ludwig Maximilians University of Munich,

Germany

\section{Key Words}

Acneiform dermatoses

Drug-induced acne

Bodybuilding acne

Gram-negative folliculitis

Acne necrotica

Acne aestivalis

\title{
Acneiform Dermatoses
}

\begin{abstract}
Acneiform dermatoses are follicular eruptions. The initial lesion is inflammatory, usually a papule or pustule. Comedones are later secondary lesions, a sequel to encapsulation and healing of the primary abscess. The earliest histological event is spongiosis, followed by a break in the follicular epithelium. The spilled follicular contents provokes a nonspecific lymphocytic and neutrophilic infiltrate. Acneiform eruptions are almost always drug induced. Important clues are sudden onset within days, widespread involvement, unusual locations (forearm, buttocks), occurrence beyond acne age, monomorphous lesions, sometimes signs of systemic drug toxicity with fever and malaise, clearing of inflammatory lesions after the drug is stopped, sometimes leaving secondary comedones. Other cutaneous eruptions that may superficially resemble acne vulgaris but that are not thought to be related to it etiologically are due to infection (e.g. gramnegative folliculitis) or unknown causes (e.g. acne necrotica or acne aestivalis).
\end{abstract}

\section{Introduction}

The term 'acneiform' refers to conditions which superficially resemble acne vulgaris but are not thought to be related to it etiologically.

Acneiform eruptions are follicular reactions beginning with an inflammatory lesion, usually a papule or pustule. Comedones are uncommon and usually follow pustules. These follicular inflammatory responses are almost always induced by drugs, but they are occasionally due to infection (e.g. gram-negative folliculitis) or unknown cause (e.g. acne necrotica or acne aestivalis).

\section{Drug-Induced Acne}

Certain drugs are capable of aggravating preexisting acne or of inducing acne fulminans and acneiform eruptions in predisposed subjects, similar in distribution to acne vulgaris but often of acute onset and predominantly inflam- matory (acne medicamentosa) [1]. The diagnosis of an acneiform eruption should be considered if the lesions are seen in an unusual localization for acne, e.g. involvement of distal parts of the extremities (table 1). In contrast to acne vulgaris, which always begins with faulty keratinization in the infundibula (microcomedones), comedones are usually absent or may develop secondarily. Sometimes signs of systemic drug toxicity such as fever and malaise are present.

The substances responsible for acneiform eruptions are corticosteroids (steroid acne) [2] (fig. 1), anabolic steroids including danazole [3] and testosterone (bodybuilding acne, doping acne), corticotropin [4], tetracyclines [5], vitamins $\mathrm{B}_{1}, \mathrm{~B}_{6}$ [6], $\mathrm{B}_{12}$ [7], and $\mathrm{D}_{2}$, anticonvulsants [8], psychopharmacologic drugs, e.g. lithium carbonate [9] or amineptine [10], isoniazid [11], quinidine [12], azathioprine [13], cyclosporine A [14], etretinate [15], penicillamine, iodides and bromides (halogen acne) [16]. Some drugs such as testosterone and anabolic drugs may even induce acne fulminans [17]. Although 13-cis-retinoic acid is

\begin{tabular}{ll}
\hline KARGER & ( 1998 S. KargerAG, Basel \\
E-Mail karger@ karger.ch & 1018-8665/98/1961-0102\$15.00/0 \\
$\begin{array}{l}\text { Fax +41 61 306 12 34 } \\
\text { www.karger.com }\end{array}$ & $\begin{array}{l}\text { This article is also accessible online at: } \\
\text { http://BioMedNet.com/karger }\end{array}$
\end{tabular}

Gerd Plewig, MD
Department of Dermatology
Ludwig-Maximilians-Universität, Frauenlobstrasse 9-11
D-80337 Munich (Germany)
Tel. 08951604601 , Fax 08951604664 
Fig. 1. Steroid acne from oral intake of prednisolone. Widespread uniform papulopustules cover most of the back, wherever there are sebaceous follicles.

Fig. 2. Steroid acne. The earliest detectable event is a focal necrosis of the midportion of the infundibulum of the sebaceous follicle. There is no comedo. Lymphocytes and some granulocytes are at the breach. Hematoxylin and eosin stain.
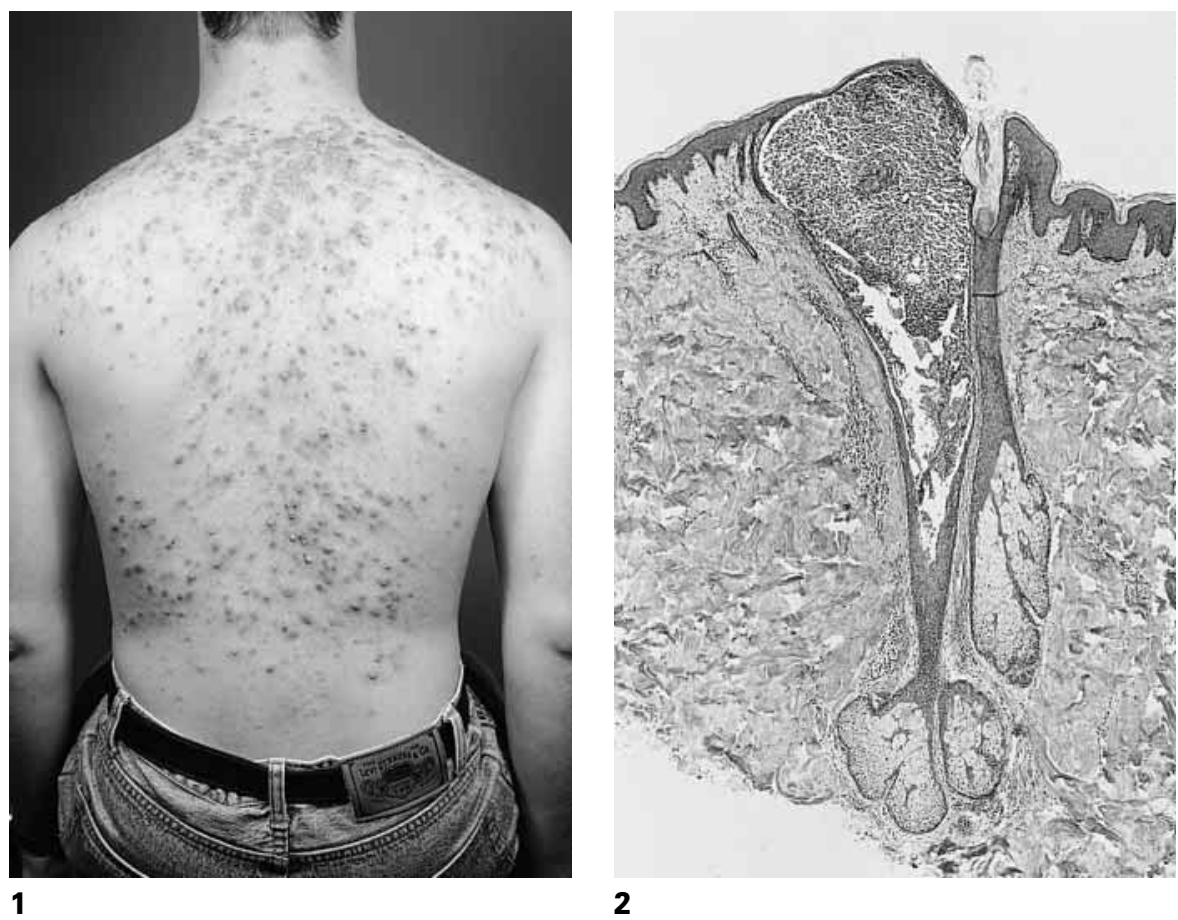

Table 1. Characteristics of drug-induced acne

Sudden onset

Widespread extent from the beginning

Monomorphous appearance

Unusual localizations for acne, e.g. involvement of distal extremities Unusual age for acne vulgaris

Exposure to drug

used in the treatment of severe acne, paradoxically this drug is reported to cause acne fulminans as an adverse effect [18]. In most cases the exact pathogenesis of acne medicamentosa remains unknown.

Acneiform eruptions are nonallergic reactions that do not appear to have a single mechanism. Therefore skin testing is not useful in diagnosing drug-induced acne. The patient's history, the clinical findings, the exposure to one of the known causative drugs and the disappearance of lesions after discontinuation of the offending medication should lead to the diagnosis.

For example, steroid acne appears to result from a direct effect of the steroid on the follicular epithelium, causing a focal degeneration with a localized intrafollicular and perifollicular neutrophilic inflammatory reaction (fig. 2-4) [2]. It is assumed that large lesions do not occur because of the anti-inflammatory effect of the steroid itself. Also,

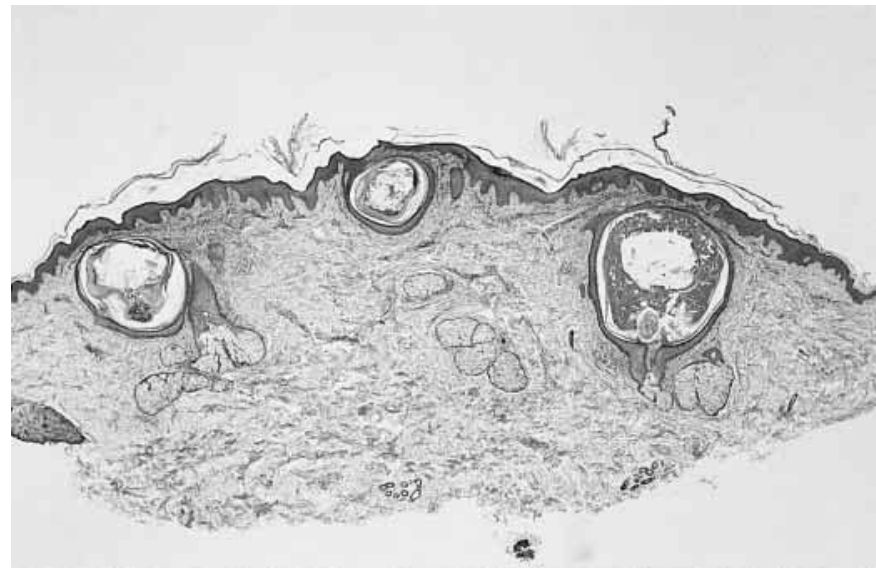

Fig. 3. Steroid acne. Advanced stage of comedo formation. This is a closed comedo, with colonization of bacteria, keratinization of the sebaceous duct and involution of the sebaceous glands. Hematoxylin and eosin stain.

unlike acne vulgaris, follicular hyperkeratosis is not an early event, as was once believed [19].

An acneiform eruption may be observed following the prolonged systemic administration of iodides or bromides $[16,20,21]$. It is important to note that halogens may be present in unsuspected sources, e.g. iodides in drugs used for the treatment of colds and asthma, radioopaque contrast materials and vitamin-mineral preparations, or bromides in 


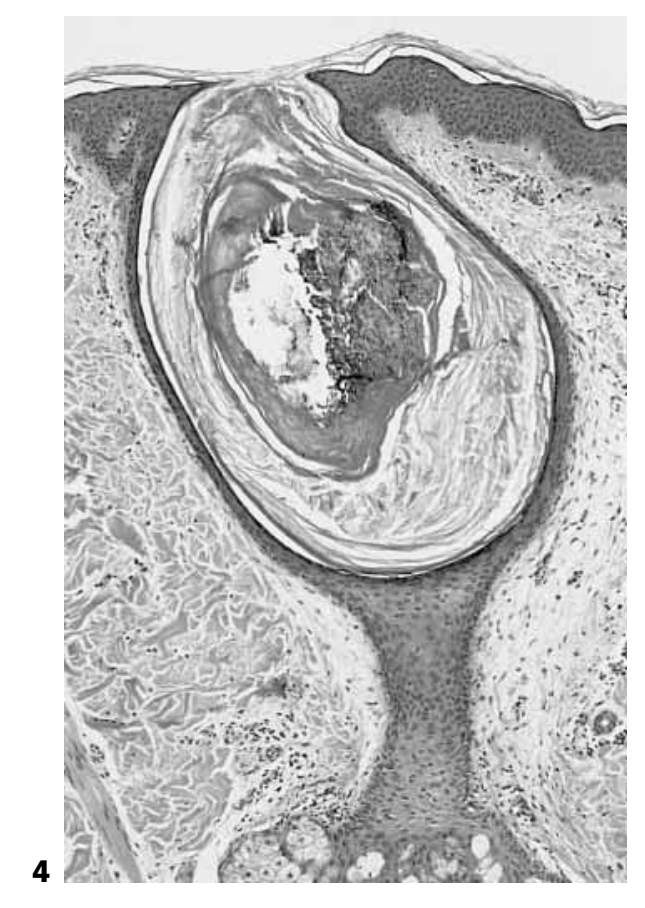

sedatives, analgesics and cold remedies. Fad diets with high iodide supplementation, such as large quantities of kelp pills [22], seem to be related to flares of preexisting acne.

Bodybuilding acne (doping acne, power athlet acne) is a popular variant of androgen-induced acne [17]. It is most common in bodybuilders and weight lifters who have a history of oral or parenteral intake of anabolic-androgenic steroids and vitamin B complexes. Often there is no history of previous acne. These patients may show mild to severe acne, even acne fulminans, oily skin and hair, androgenic alopecia of the scalp, hirsutism of the face and body, and gynecomastia. Extracutaneous side effects of anabolic-androgenic steroids, e.g. changes in libido, increased aggressiveness, mood swings (euphoria, depression, delusions), liver disease (peliosis or cholestatic hepatitis, liver tumors) or cardiovascular disorders (e.g. cardiomyopathy) may also be present.

Drugs which cause acneiform eruptions can, at the same time, aggravate a preexisting acne. In these cases, underlying acne with a sudden worsening is mixed with additional crops of acneiform eruptions.

\section{Gram-Negative Folliculitis}

This is a follicular pyoderma and not a variant of acne, but it is a characteristic complication of long-term oral antibiotic acne treatment $[23,24]$. Gram-negative folliculitis

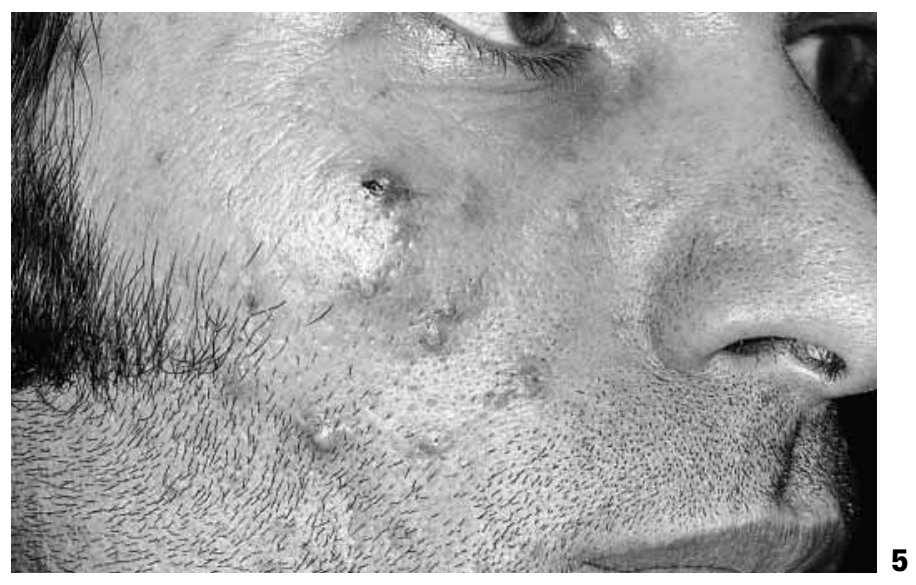

Fig. 4. Steroid acne. Later stage of a secondary comedo following an initial follicular rupture. Asymmetry is an important clue. Hematoyxlin and eosin stain.

Fig. 5. Type I gram-negative folliculitis is caused by a variety of gram-negative organisms. Papules and pustules are found particularly around the nose, mouth and cheeks on extremely oily skin.

occurs in patients who have had moderately inflammatory acne for long periods and have been treated with long-term antibiotics, especially tetracyclines. At first antibiotics, mainly tetracyclines, provide adequate control. Later on, the drug not only becomes ineffective but actually makes the condition worse.

Two types of gram-negative folliculitis have been described [25]. While on antibiotic treatment, patients develop either papules and papulopustules 3-6 $\mathrm{mm}$ in diameter flaring out from the anterior nares (type I; fig. 5) or, on rare occasions, fluctuant, deep-seated, acne-conglobata-like nodules (type II). Culture of these lesions usually reveals a species of Enterobacter, Klebsiella or Escherichia coli (type I) or, from the deep cystic lesions, Proteus (type II). With long-term antibiotic therapy the anterior nares may become colonized with these gram-negative organisms. Immunological factors may contribute to the pathogenesis of this disease [26]. Organisms are often scarce and their detection may require sampling several lesions. Problems often arise in establishing a correct microbiological diagnosis. Inadequate sampling, dried-out swabs and long delay between culturing the pustules and arrival of the specimen in the laboratory are factors which may account for this.

Systemic treatment with isotretinoin $(0.5-1.0 \mathrm{mg} / \mathrm{kg}$ daily) for $4-5$ months is usually effective $[27,28]$. The mode of action of isotretinoin is indirect. Gram-negative organisms are wet germs and normally require abundant moisture for survival. Isotretinoin dries out the skin, taking 
away this essential factor for the survival of these bacteria. This also happens in the mucous membranes but not to the same degree. In selected patients, oral antibiotics (cephalosporins) for 2 weeks may have to be combined with isotretinoin, which is given for several months. Simultaneous colonization of the urogenital tract, mostly without clinical symptoms such as prostatitis, with gram-negative organisms has been detected in some of our patients. Oral isotretinoin alone does not seem to cure these infections. Particularly in these patients isotretinoin plus an oral antibiotic seem appropriate.

\section{Acne necrotica}

Acne necrotica (synonyms are acne necrotica miliaris, acne varioliformis, acne frontalis, acne atrophica, pustular perifolliculitis, necrotizing lymphocytic folliculitis) is an uncommon papulopustular, follicular eruption that eventually leaves depressed superficial scars resembling smallpox scars, hence the previous term acne varioliformis [29]. The disease occurs after puberty without sexual predilection.

The etiology is essentially unknown. Although coagulase-positive staphylococci have been cultured from the pustules, the importance of this organism is difficult to assess. Propionibacterium acnes has also been implicated [30]. Some clinicians doubt the existence of this condition as a distinct entity.

The process begins as a brown-red, grouped, papulopustular perifollicular lesion (fig.6). As the pustules increase in size, their centers become depressed and a dry crust develops that is frequently hemorrhagic. This is the papulonecrotic lesion. The resulting scar is also depressed and varioliform. If the lesions are recurrent, the resulting scar may be reticulated. The typical distribution and morphology of scarring is a lifelong evidence of previous acne necrotica. Lesions are located most often at the temporal area of the scalp [31] but have been described on the nose, cheek, chest and back. Patients with the disease often suffer from seborrhea. The folliculitis is frequently accompanied by intense itching.

The earliest lesion is an intraepidermal vesicle filled with serum [32]. Later, a superficial follicular pustule filled with polymorphonuclear leukocytes is found (fig. 7). At the opening of the follicle a crust made up of serum and clumps of bacteria may be present. A lymphocytic infiltrate occurs limited to the perifollicular area, and some neutrophils may be seen migrating the follicular wall. Healing with fibrosis and scar formation often results [33].

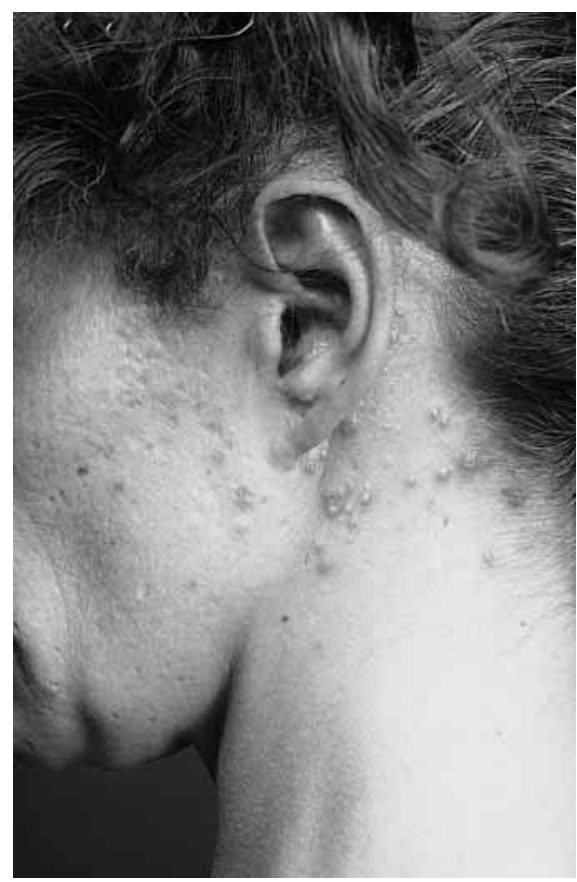

Fig. 6. Acne necrotica. Typical distribution pattern along the hairline, with widespread involvement of the hairy scalp. Scarring on the forehead is a result of previous acne.

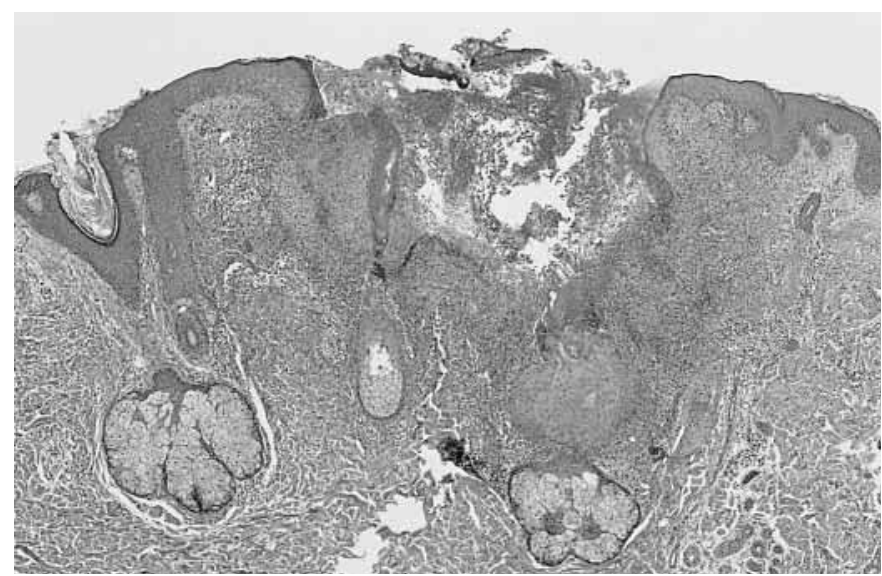

Fig. 7. Acne necrotica. A wedge-shaped central necrosis, totally obscuring the original follicle, prevails. A parakeratotic cap sits on top of a fibrinoid necrosis. The lymphocytic infiltrate is dense, stretching out bilaterally to cause prominent spongiosis of epidermis and neighboring follicles. Hematoxylin and eosin stain. 
Bacterial cultures and sensitivity studies are generally not helpful as treatment guides. Routine aerobic cultures invariably grow Staphylococcus epidermidis or sometimes Staphylococcus aureus. Gram staining may demonstrate numerous intracellular and extracellular gram-positive pleomorphic rods compatible with $P$. acnes.

Systemic antibiotics as for acne should be initiated $[34,35]$. Tetracycline, $250 \mathrm{mg}$ four times daily, or erythromycin in a similar dosage is appropriate. In our experience, a therapeutic regimen including isotretinoin $(0.5-1.0 \mathrm{mg} / \mathrm{kg})$ combined with corticosteroids, e.g. $0.5-1.0 \mathrm{mg} / \mathrm{kg}$ of prednisolone, may also be effective. Additional measures such as antibacterial washes, e.g. chlorhexidine or hexachlorophene, and warm wet compresses to loosen the crusts may be helpful. A topical corticosteroid lotion or aerosol helps alleviate pruritus. Treatment must usually be prolonged for weeks or months. Once control has been achieved, antibacterial lotions such as clindamycin $1 \%$ or erythromycin $2 \%$ may be substituted for systemic antibiotics or isotretinoin. Daily shampooing, the use of antibacterial cleansers and topical antibiotic therapy may be of value for prophylaxis.

\section{Acne aestivalis}

Acne aestivalis (Mallorca acne) refers to a monomorphous eruption, consisting of multiple papular lesions, which has been reported to develop after sun exposure (fig. 8). Hjorth et al. [36] referred to this as Mallorca acne since it occurred in many Scandinavians after they had been on a sunny vacation in Mallorca after a long dark winter. Although the cheeks may be involved, the lesions commonly extend onto the sides of the neck, chest, shoulders and upper arms. The last location is rare in ordinary acne. Histologically, the lesions resemble steroid acne in that they show a focal follicular destruction with neutrophilic infil-

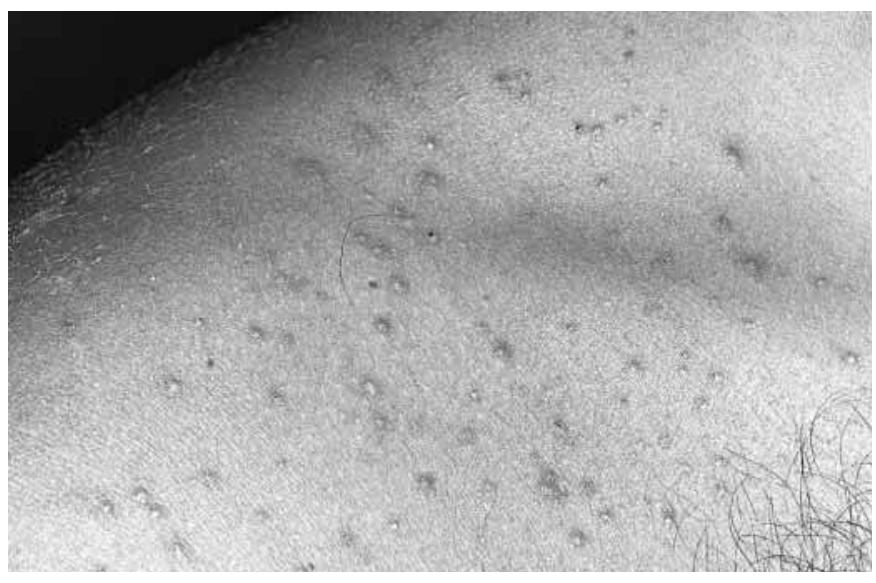

Fig. 8. Acne aestivalis. The lesions are monomorphic, follicular papules containing a small central core of horn with a surrounding inflammatory reaction.

trate. Comedones are notably not apparent as are pustules. The disease may abate after many months, probably because of less intensive sunbathing. The eruption is due to the effects of UV radiation, mainly UVA. Similar follicular eruptions have also been reported as an unusual side effect of photochemotherapy (PUVA) [37]. Like patients with polymorphous light reaction, subjects prone to acne aestivalis react the same way year after year. Indeed, there is some evidence that this is a papular variant of polymorphous light eruption.

While oral antibiotics are ineffective, topical tretinoin or benzoyl peroxide is valuable in bringing about resolution of lesions. Topical corticosteroids should be avoided, since they may aggravate the disease. Prophylaxis can be offered by gradually increasing exposure to full-body artificial UV radiation, UVB or UVA, either alone or in combination, or even PUVA therapy prior to the first exposure to natural sunlight.

\section{References}

1 Bedane C, Souyri N: Les acnés induites. Ann Dermatol Vénéréol 1990;117:53-58.

2 Hurwitz RM: Steroid acne. J Am Acad Dermatol 1989;21:1179-1181.

3 Greenberg RD: Acne vulgaris associated with antigonadotropic (danazol) therapy. Cutis 1979;24:431-432.

4 Sullivan M, Zeligman I: Acneform eruption due to corticotropin. Arch Dermatol 1956;73: 133-141.
5 Bean SF: Acneiform eruption from tetracycline. Br J Dermatol 1971;85:585-586.

6 Sherertz EF: Acneiform eruption due to 'megadose' vitamins $\mathrm{B}_{6}$ and $\mathrm{B}_{12}$. Cutis 1991; 48:119-120.

7 Dupré A, Albarel N, Bonafe JL, Christol B, Lassere J: Vitamin B-12 induced acnes. Cutis 1979;24:210-211.

8 Greenwood R, Fenwick PBC, Cunliffe WJ: Acne and anticonvulsants. Br Med J 1983;287: 1669-1670.
9 Kanzaki T: Acneiform eruption induced by lithium carbonate. J Dermatol 1991;18: 481-483.

10 Vexiau P, Gourmel B, Castot A, Husson C, Rybojad M, Julien R, Fiet J, Hardy N, Puissant A, Cathelineau G: Severe acne due to chronic amineptine overdose. Arch Dermatol Res 1990;282:103-107. 
11 Cohen LK, George W, Smith R: Isoniazidinduced acne and pellagra: Occurrence in slow inactivators of isoniazid. Arch Dermatol 1974; 109:377-381.

12 Burkhart CG: Quinidine-induced acne. Arch Dermatol 1981;117:603-604.

13 Schmoeckel C, Von Liebe V: Akneiformes Exanthem durch Azathioprin. Hautarzt 1983; 34:413-415.

14 Bencini PL, Montagnino G, Crosti C, Sala F, DeVecchio A: Acne in a kidney transplant patient treated with cyclosporin A. Br J Dermatol 1986;114:396-397.

15 Lowe L, Herbert AA: Cystic and comedonal acne: A side effect of etretinate therapy. Int $\mathrm{J}$ Dermatol 1989;28:482.

16 Hitch JM: Acneform eruptions induced by drugs and chemicals. JAMA 1967;200: 879-880.

17 Heydenreich G: Testosterone and anabolic steroids and acne fulminans. Arch Dermatol 1989;125:571-572.

18 Choi EH, Bang D: Acne fulminans and 13-cisretinoic acid. J Dermatol 1992;19:378-382.

19 Van Scott EJ, MacCardle C: Keratinization of the duct of the sebaceous gland and growth of the hair follicle in the histogenesis of acne in human skin. J Invest Dermatol 1956;27: 405-413.
20 Manok M: Zur Pathogenese der Bromakne. Dermatologica 1963;127:375-380.

21 Soper LE, Vitez TS, Weinberg D: Metabolism of halogenated anesthetic agents as a possible cause of acneiform eruptions. Anesth Analg 1973;52:125-127.

22 Harrell BL, Rudolph AH: Kelp diet: A cause of acneiform eruption. Arch Dermatol 1976;112: 560.

23 Fulton JE, McGinley K, Leyden JJ, Marples R: Gram-negative folliculitis in acne vulgaris. Arch Dermatol 1968;98:349-353.

24 Plewig G, Braun-Falco O: Gramnegative Follikulitis. Hautarzt 1974;25:541-546.

25 Leyden JJ, Marples RR, Mills OH Jr, Kligman AM: Gram-negative folliculitis - A complication of antibiotic therapy in acne vulgaris. $\mathrm{Br} \mathrm{J}$ Dermatol 1973;88:533-538.

26 Neubert U: Immunabweichungen bei sogenannter Gramnegativer Follikulitis. Hautarzt 1983;34(suppl 6):277-278.

27 Neubert U, Plewig G, Ruhfus A: Treatment of gram-negative folliculitis with isotretinoin. Arch Dermatol Res 1986;278:307-313.

28 Plewig G, Nikolowski J, Wolff HH: Action of isotretinoin in acne, rosacea and gram-negative folliculitis. J Am Acad Dermatol 1982;6: 766-785.
29 Milde P, Goerz G, Plewig G: Acne necrotica (varioliformis). Nekrotisierende lymphozytäre Follikulitis. Hautarzt 1993;44:34-36.

30 Maibach H: Scalp pustules due to Corynebacterium acnes. Arch Dermatol 1967;96: 453-455.

31 Lane JE: Acne necrotica miliaris of the scalp. Arch Dermatol Syphilol 1933;28:10-12.

32 Montgomery H: Acne necrotica miliaris of the scalp: A clinical and histological study. Arch Dermatol Syphilol 1937;36:40-44.

33 Kossard S, Collins A, McCrossin I: Necrotizing lymphocytic folliculitis: The early lesion of acne necrotica. J Am Acad Dermatol 1987;16: 1007-1014.

34 Hersle K, Mobacken H, Moller A: Chronic non-scarring folliculitis of the scalp. Acta Derm Venereol (Stockh) 1979;59:249-253.

35 Stritzler C, Friedman R, Loveman AB: Acne necrotica: Relation to acne necrotica miliaris and response to penicillin and other antibiotics. Arch Dermatol Syphilol 1951;64:464-469.

36 Hjorth N, Sjolin KE, Sylvest B, Thomsen K: Acne aestivalis - Mallorca acne. Acta Derm Venereol (Stockh) 1972;52:61-63.

37 Nielson EB, Thorman J: Acne-like eruptions induced by PUVA treatment. Acta Derm Venereol (Stockh) 1978;58:374-375. 